\section{An alternative endoscopic treatment for massive gastric bezoars: Ho:YAG laser fragmentation}

Gastrointestinal (GI) bezoars are aggregates of indigestible material that occur mainly in patients with altered GI anatomy (i.e. bariatric surgery, partial gastrectomy) and/or motility disorders (i.e. gastroparesis, drug-induced delayed gastric emptying). Other risk factors are high fiber intake, psychiatric diseases, and severe constipation [1].

Treatment strategies for bezoar fragmentation include pharmacological, endoscopic, and surgical approaches. Endoscopic treatment of gastric bezoar consists of attempted fragmentation by using different devices such as snares, forceps, baskets, lithotripters, argon plasma coagulation, and needle-knife [2,3]. The use of a laser-ignited mini-explosive technique with neodymium-doped yttrium aluminium garnet (Nd:YAG) laser has also been described [4]. To date, only one article from China (original language) has described the use of holmium:YAG (Ho: YAG) laser lithotripsy in this field [5].

We present two video cases of massive gastric phytobezoar, which were irresponsive to pharmacological approaches and were successfully treated with Ho: YAG laser (Lumenis Ltd., Yokneam, Israel). The first patient was a 73-year-old man with a history of Billroth I partial gastrectomy for gastric cancer, who had endoscopic evidence of a massive, hard, phytobezoar, $10 \mathrm{~cm}$ in diameter $($ Video 1$)$. The phytobezoar was successfully treated with 200 micron laser fiber (Lumenis VersaPulse P20 lithotripter, frequency $4-8 \mathrm{~Hz}$, pulse energy $2000 \mathrm{~mJ}$ ) The procedure time was 60 minutes.

The second patient was a 58-year-old woman with a gastric band for treatment of obesity, who showed a large phytobezoar $(8 \mathrm{~cm}$ in diameter) in the proximal gastric pouch ( Video 2 ). In this case, we used a 550 micron laser fiber, which allowed the diffusion of higher pulse ener- gy (Lumenis VersaPulse PowerSuite 100W lithotripter, $10-30 \mathrm{~Hz}, 3000 \mathrm{~mJ}$ ), resulting in a shorter procedure time (24 minutes). In both procedures, complete bezoar fragmentation was achieved in a single endoscopy session.

No postprocedural complications were registered in the patients and they were discharged 1 day after the endoscopic treatment.

In conclusion, Ho:YAG laser fragmentation represents a safe, effective, and rapid endoscopic approach for the treatment of massive gastric bezoars.

Endoscopy_UCTN_Code_TTT_1AO_2AL

Competing interests: None

\section{Giuseppe Grande, Mauro Manno, Claudio Zulli, Carmelo Barbera, Santi Mangiafico, Nadia Alberghina, Rita Luisa Conigliaro}

Gastrointestinal Endoscopy Unit, NOCSAE Baggiovara Hospital, Modena, Italy

\section{References}

1 Iwamuro M, Okada $\mathrm{H}$, Matsueda $\mathrm{K}$ et al. Review of the diagnosis and management of gastrointestinal bezoars. World J Gastrointest Endosc 2015; 7: 336-345

2 Kuo JY, Mo LR, Tsai CC et al. Nonoperative treatment of gastric bezoars using electrohydraulic lithotripsy. Endoscopy 1999: 31; $386-388$

3 Iwamuro M, Tanaka S, Shiode J et al. Clinical characteristics and treatment outcomes of nineteen Japanese patients with gastrointestinal bezoars. Intern Med 2014; 53: 1099-1105

4 Mao Y, Qiu H, Liu Q et al. Endoscopic lithotripsy for gastric bezoars by Nd:YAG laserignited mini-explosive technique. Lasers Med Sci 2014; 29: $1237-1240$

$5 \mathrm{Xu}$ C, Li PY, Qin H et al. Curative effect of endoscopic holmium laser lithotripsy in management of gastric bezoars. World Chinese J Digestol 2015; 23: 3277

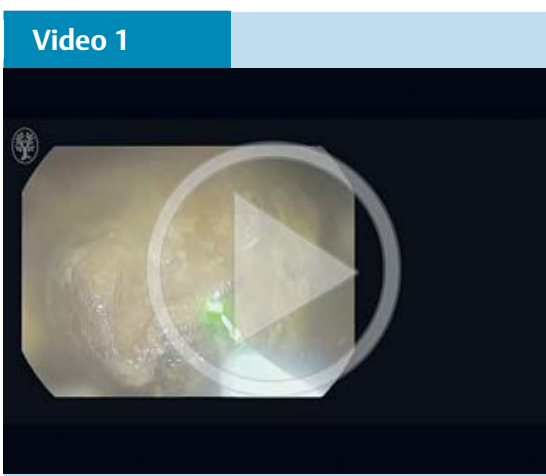

Holmium:YAG laser treatment (frequency $4-8 \mathrm{~Hz}$, pulse energy $2000 \mathrm{~mJ}$ ) of a $10-\mathrm{cm}$ phytobezoar in a 73-year-old man with Billroth I anatomy (procedure time 60 minutes).

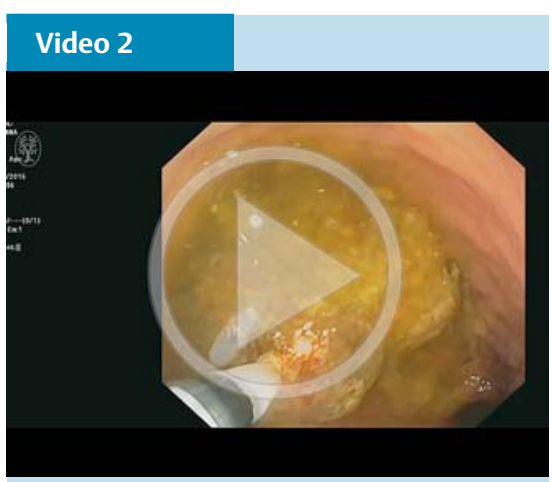

Holmium:YAG laser treatment (Lumenis VersaPulse PowerSuite $100 \mathrm{~W}$ lithotripter, $10-30 \mathrm{~Hz}$, $3000 \mathrm{~mJ})$ of an $8-\mathrm{cm}$ phytobezoar in the proximal gastric pouch of a 58-year-old woman with a gastric band (procedure time 24 minutes).

Bibliography

DOI http://dx.doi.org/

10.1055/s-0042-109057

Endoscopy 2016; 48: E217

(c) Georg Thieme Verlag KG

Stuttgart · New York

ISSN 0013-726X

\section{Corresponding author}

Giuseppe Grande, MD

Gastrointestinal Endoscopy Unit

NOCSAE Hospital

via Pietro Giardini 1355

Baggiovara di Modena 41126

Italy

Fax: +39-059-3961216

giuseppegrande1984@gmail.com 